\title{
Effects of Maternal Starvation on Hepatocyte Proliferation in the Late Gestation Fetal Rat
}

\author{
PHILIP A. GRUPPUSO, JOAN M. BOYLAN, PADMANABHAN ANAND, AND \\ THERESA C. BIENIEKI
}

\author{
Department of Pediatrics, Rhode Island Hospital and Brown University, Providence, Rhode Island 02903
}

\section{ABSTRACT}

\begin{abstract}
Fetal growth retardation, a common end point for a variety of conditions affecting mother and fetus, is associated with reduced liver mass. We have performed studies to determine the mechanism for decreased liver mass in a maternal starvation model of fetal growth restriction in the rat. Pregnant dams were deprived of food for $48 \mathrm{~h}$ before delivery on embryonic day 19 (E19). Fetal body weight was not affected. However, fetal liver weight was reduced by approximately $15 \%$. Immunostaining of fetal liver for proliferating cell nuclear antigen and flow cytometry on isolated fetal hepatocytes showed G1 cell cycle arrest in samples from starved dams. Based on our prior studies showing attenuated hepatic insulin signaling in the late gestation fetal rat, we tested the hypothesis that G1 arrest in our model might be due to altered nutrient signaling. Fetal plasma amino acid analyses showed no decrease in branched-chain amino acids, but arginine concentrations were decreased in fetuses of fasted mothers. Reduced arginine in E19 fetal hepatocyte culture media was associated with decreased DNA synthesis. Whereas levels of
\end{abstract}

cyclins D and $\mathrm{E}$ were unchanged in fetal hepatocytes exposed to low arginine, cyclin E-dependent kinase activity was reduced. Low arginine also induced changes in the translational machinery, indicative of impaired signaling through the nutrient sensing kinase mammalian target of rapamycin. Our results are consistent with the hypothesis that restricted nutrient availability signals to the hepatocyte cell cycle in fetuses of fasted mothers, thereby accounting for decreased hepatocyte proliferation and liver mass. (Pediatr Res 57: 185-191, 2005)

\begin{tabular}{l}
\multicolumn{1}{c}{ Abbreviations } \\
CKI, cyclin-dependent kinase inhibitor \\
DAPI, 4',6-diamidino-2-phenylindole \\
E19, embryonic day 19 \\
MEM $\boldsymbol{\alpha}$, minimal essential media $\alpha$ \\
mTOR, mammalian target of rapamycin \\
PCNA, proliferating cell nuclear antigen \\
TUNEL, TdT-mediated dUTP nick-end labeling
\end{tabular}

Impaired fetal somatic growth is a frequent occurrence and a common end point for a spectrum of disorders affecting the mother and fetus. Given that hepatic growth and functional differentiation are required for normal metabolic homeostasis during the perinatal period, the liver represents an important target for the various mechanisms that can limit fetal growth. In the rat, the latter portion of gestation is a period of time when hepatocytes proliferate at a high rate $(1,2)$, resulting in a marked increase in liver mass over the last several days of gestation. A variety of rat models, all of which are associated with retardation of fetal somatic growth, have been shown to be associated with impaired hepatic growth (3-5). This association may be a consequence of impaired hepatocyte proliferation, decreased cell size as a function of impaired cell

Received April 19, 2004; accepted July 28, 2004.

Correspondence: Philip A. Gruppuso, M.D., Department of Pediatrics, Rhode Island Hospital, 593 Eddy St., Providence, RI 02903; e-mail: Philip_Gruppuso@brown.edu

Supported by United States Public Health Service grants HD24455, HD35831, and DK59815, and by the Rhode Island Hospital Department of Pediatrics Research Endowment Funds.

DOI: 10.1203/01.PDR.0000151646.55587.0F growth, increased cell loss via apoptosis, or a combination of all three factors.

Insulin has long been assigned a key role in the regulation of fetal somatic growth, with fetal hyperinsulinemic and hypoinsulinemic states showing parallel changes in somatic and hepatic growth (6). Although this has been inferred as resulting from the direct effects of insulin on the developing liver, our previous studies aimed at understanding the control of hepatocyte proliferation in the late gestation rat have indicated a very limited role for insulin in the regulation of the growth of these cells $(7,8)$. This raises the likelihood that signaling mechanisms other than those downstream from the insulin receptor are involved in cell cycle regulation and cell growth under conditions of limited fetal growth. A candidate mechanism involves the mammalian target of rapamycin, mTOR, a key regulator of cell growth (9).

TOR kinases, originally identified in yeast as the cellular targets of the immunosuppressant rapamycin are members of the phosphatidylinositol 3-kinase superfamily of protein kinases (9). They provide a link between nutrient availability and cell growth (10). The mammalian homologue of yeast TOR, 
mTOR, can regulate cell size through the control of protein translation, ribosomal biogenesis, and autophagy (9). mTOR can also regulate cell proliferation, apoptosis, and differentiation through transcriptional and translational effects on a broad spectrum of targets (9). Downstream effectors of mTOR include protein kinases that phosphorylate ribosomal protein S6, and the inhibitory protein of the eukaryotic initiation factor eIF4E, termed 4E-BP1 (9). Finally, mTOR is the sole known mammalian target for rapamycin, which potently inhibits its ability to transduce a signal.

The factors described above, a limited role for insulin and a possible direct role for nutrient signaling, led us to hypothesize that impaired hepatic growth under circumstances of limited fetal growth in the rat might be associated with decreased nutrient signaling through mTOR. We chose to test this hypothesis in a well-established rat model of attenuated fetal growth, maternal starvation $(4,11)$.

\section{MATERIALS AND METHODS}

Animals. Except where noted, all studies were carried out in timed pregnant Sprague-Dawley rats (Charles River Laboratories, Wilmington, MA). Control fetuses were derived from animals that were fed standard laboratory chow ad libitum. Fetuses of fasted mothers were delivered after $48 \mathrm{~h}$ of maternal starvation (free access to water). All fetuses were delivered on E19, term being $21 \mathrm{~d}$. Cesarean section was performed under pentobarbital sodium anesthesia $(50 \mathrm{mg} / \mathrm{kg}$ i.p.). Fetuses were rapidly weighed, then subjected to laparotomy to remove the entire liver. Liver tissue to be used for preparation of homogenates was flash frozen in liquid nitrogen and stored at $-70^{\circ} \mathrm{C}$ until use. Where noted, livers were used immediately for preparation of hepatocytes.

Plasma for amino acids was collected from fetuses at the time of cesarean section as described previously (11). Plasma obtained from the first six fetuses per litter was pooled and analyzed as a single sample by ion exchange chromatography (12).

Liver samples from adult rats were obtained as previously described (7) from males weighing between 150 and $175 \mathrm{~g}$. Tissue for direct analysis was flash frozen in liquid nitrogen and held at $-70^{\circ} \mathrm{C}$ until preparation of homogenates.

All animal studies were approved by the Rhode Island Hospital Institutional Animal Care and Use Committee.

Hepatocyte preparation and culture. Fetal hepatocytes were isolated, plated, and maintained in culture under defined conditions as described previously (1). Hepatocytes were plated onto untreated Primaria plasticware $(2 \times$ $10^{6}$ cells per $100 \mathrm{~mm}$ plate) using supplemented (1) MEM $\alpha$ (Invitrogen, Carlsbad, CA) containing 5\% fetal bovine serum. After a 2-h attachment period, the culture medium was changed to supplemented MEM $\alpha$ without serum. Where noted, custom MEM $\alpha$ lacking glucose, leucine, arginine, and glutamine (also obtained from Invitrogen) was used after repletion of selected nutrients. The levels of these nutrients in the selectively deficient media is expressed as a percentage of the usual concentrations in MEM $\alpha: 5.6 \mathrm{mM}$ glucose, $0.4 \mathrm{mM}$ leucine, $1.66 \mathrm{mM}$ arginine, and $0.5 \mathrm{mM}$ glutamine. The $\operatorname{MEM} \alpha$ that we used throughout these studies is a relatively simple, sodium bicarbonate-based medium that we supplement with $1 \mathrm{mM}$ proline, $0.2 \mathrm{mM}$ serine, $0.2 \mathrm{mM}$ aspartate, $1 \mathrm{mM}$ pyruvate, and $1 \mu \mathrm{g} / \mathrm{mL}$ hydrocortisone. This formulation was previously found to maximally support fetal hepatocyte proliferation in vitro (1)

Where noted, freshly isolated fetal hepatocytes were subjected to flow cytometry, as performed previously (13).

Immunohistochemical and immunofluorescent analyses. Detection of PCNA in sectioned, formalin-fixed liver used immunohistochemical detection with peroxidase-conjugated secondary antibody and 3,3'-diaminobenzidine as substrate (2). Immunofluorescence on cultured hepatocytes used previously described fixation methods (14) and mounting medium that contains DAPI, which allowed detection of nuclei. Image analysis of DAPI staining was used to assess liver sections for total cell number per unit area. The occurrence of apoptosis in fetal hepatocyte cultures was assessed by TUNEL. Reagents for this analysis (DeadEnd Fluorometric TUNEL System) were obtained from Promega (Madison, WI). Based on our own unpublished observations, $10 \mathrm{pM}$ transforming growth factor- $\beta$ (TGF- $\beta$ ) was used as a positive control for TUNEL staining. Confirmatory studies were performed using a cell viability assay that employs dual fluorescent dyes (LIVE/DEAD Reduced Biohazard Viability/Cytotoxicity assay, Molecular Probes, Eugene, OR).

Measurement of hepatocyte DNA synthesis. DNA synthesis was measured using incorporation of ${ }^{3} \mathrm{H}$-thymidine or immunofluorescent detection of 5-bromo-2'-deoxyuridine (BrdU). The methods used for both have been described previously $(2,14)$.

Cell cycle and translational control analyses. Liver homogenates for Western blotting were prepared as described previously (7) using the following buffer: $50 \mathrm{mM}$ HEPES, pH 7.5, $150 \mathrm{mM} \mathrm{NaCl}, 25 \mathrm{mM} \beta$-glycerophosphate, 1 $\mathrm{mM} \mathrm{NaF}, 1 \mathrm{mM}$ EDTA, $2.5 \mathrm{mM}$ EGTA, $1 \mathrm{mM} \mathrm{NaVO}, 1 \mathrm{mM}$ DTT, 0.1\% Tween-20, and $10 \%$ glycerol plus protease inhibitors $(10 \mu \mathrm{g} / \mathrm{mL}$ leupeptin, 10 $\mu \mathrm{g} / \mathrm{mL}$ aprotinin, and $34.4 \mu \mathrm{g} / \mathrm{mL} 4$-[2-aminoethyl] benzenesulfonyl fluoride). The same buffer was used for preparation of hepatocyte lysates. Protein determinations on all samples were made using the Bradford protein assay (Bio-Rad, Hercules, CA) with BSA as the standard.

Western immunoblotting used methods routinely used in our laboratory $(7,15)$. Antibody reagents for Western immunoblot detection of cell cycle components were obtained from the following sources. Rabbit polyclonal antisera directed toward cyclin E and CDK2 were obtained from Santa Cruz Biotechnology (Santa Cruz, CA). Anti-cyclin D3 was obtained from NeoMarkers (Freemont, CA). Antibodies directed toward phospho-Rb (Ser780) and phospho-Rb (Ser807/811) were purchased from Cell Signaling Technology (Beverly, MA). Anti-Rb was from BD PharMingen (San Diego, CA).

The methods used for immunoprecipitation of cyclin $\mathrm{E}$ and the direct measurement of cyclin E-associated kinase activity followed methods that we previously used for similar studies on cyclin D1-containing complexes (13).

Western immunoblotting to assess the phosphorylation state of ribosomal protein S6 was performed as described previously (7). The activation status of the translational initiation complex containing eIF4E and 4E-BP1 was measured using affinity purification with ${ }^{7}$ methyl-GTP-Sepharose 4B beads. Hepatocytes were lysed in a buffer containing $50 \mathrm{mM}$ Tris $\mathrm{HCl}, \mathrm{pH} 7.4,50 \mathrm{mM} \mathrm{KCl}$, $1 \mathrm{mM}$ EDTA, $0.5 \% \mathrm{NP}-40$, and $1 \mu \mathrm{M}$ microcystin plus the protease inhibitors described above. The lysate was incubated on ice for $30 \mathrm{~min}$ then centrifuged at $10,000 \mathrm{~g}$ at $4^{\circ} \mathrm{C}$. Supernatant protein $(275 \mu \mathrm{g})$ was incubated with $25 \mu \mathrm{L}$ of the Sepharose beads overnight at $4^{\circ} \mathrm{C}$ on a rotator. The beads were washed three times with the lysis buffer plus $100 \mu \mathrm{M}$ GTP, following which they were boiled in one volume of electrophoresis sample buffer. The resulting sample was analyzed by Western immunoblotting for eIF4E and 4E-BP1 (7).

Data analysis. Where combined data are shown, they are expressed as the mean plus one SD. Assessment of differences between groups used unpaired $t$ tests or, where noted, determination of the $95 \%$ confidence interval for the control group.

\section{RESULTS}

Maternal starvation for $48 \mathrm{~h}$ before cesarean delivery on E19 was associated with a $10 \%$ decrease in maternal weight $(281 \pm$ 7 versus $310 \pm 16 \mathrm{~g}$ ). There was no significant reduction in fetal weight (Fig. 1A). However, liver weight and the liver-tocarcass weight ratio were reduced by approximately $15 \%$ (Fig. $1, B$ and $C$ ). Microscopic morphometry on DAPI-stained liver sections (Fig. 1D) revealed no change in cell number per unit area, indicating that the decrease in liver weight was a function of a decrease in cell number, not cell size. Based on this result, we sought to determine the effect of maternal starvation on fetal hepatocyte cell cycle kinetics.

Liver cryosections from fetuses of control and fasted mothers were analyzed for PCNA expression. The number of PCNA-positive cells per unit area was reduced by about one third in fetuses of fasted mothers (Fig. 2A), indicating that maternal fasting is associated with reduced fetal hepatocyte proliferation. To determine whether or not this change could be 
A

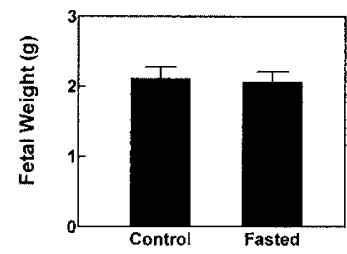

B

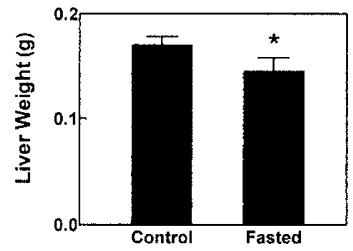

C

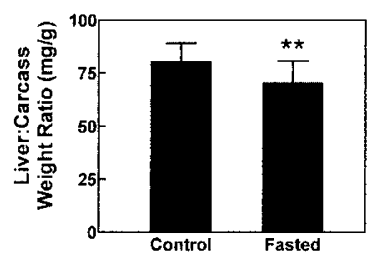

D

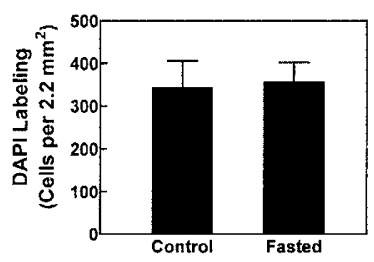

Figure 1. The effect of $48 \mathrm{~h}$ maternal fasting on fetal body weight and liver weight. Mothers were either fed ad libitum or fasted for $48 \mathrm{~h}$ before cesarean delivery on E19. Fetal weight $(A)$, fetal liver weight $(B)$, the liver weight to body weight ratio $(C)$, and nuclear density (DAPI staining, $D$ ) are shown as the mean $+1 \mathrm{SD}$ for each group. The data represent analysis of the means for each of four litters per condition. $* p<0.02 ; * * p<0.03$.

A

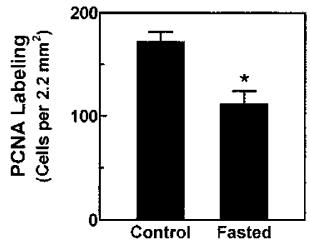

B

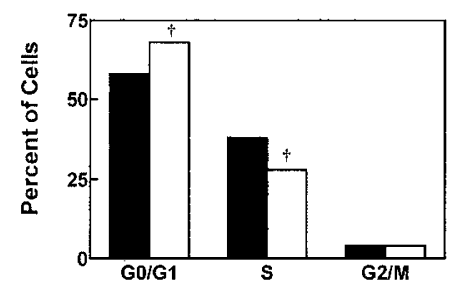

Figure 2. Cell cycle analysis of fetal livers and hepatocytes from control vs fasted mothers. (A) Liver sections were analyzed for PCNA labeling. Data are shown as the mean $+1 \mathrm{SD}$ for three livers per group. *Significant difference between groups ( $p<0.001 ; n=3$ per group). (B) Fetal hepatocytes were isolated from a control dam (solid bar) and a fasted dam (unfilled bar) and analyzed by flow cytometry. $†$ Results that were outside the $95 \%$ confidence limits for the corresponding control group.

accounted for by arrest in the G1 phase of the cell cycle, hepatocytes isolated from control or fasted mothers were analyzed by flow cytometry. Results (Fig. 2B) confirmed a modest but significant increase in the proportion of cells in G1 and a decrease in $\mathrm{S}$ in the preparations from fasted mothers.

Based on this result, we undertook an analysis of G1 cell cycle components using whole liver homogenates prepared from fetuses of control and fasted mothers. Homogenates were subjected to Western blotting for cyclin $\mathrm{E}$, phosphorylated $\mathrm{Rb}$, and total $\mathrm{Rb}$. Homogenates were also analyzed for cyclin E-dependent kinase activity. The results of these experiments (not shown) did not demonstrate any differences between the two groups. We therefore proceeded to correlative in vitro studies.

Our approach to the in vitro studies was to identify candidate nutrients that might alter fetal hepatocyte cell cycle activity. We focused on circulating amino acids. Plasma from fetuses of control and starved mothers were subjected to quantitative amino acid analysis. Results (Fig. 3) showed modest but significant changes in several amino acids. Maternal starvation was associated with decreases in fetal plasma threonine, arginine and proline concentrations. Lysine concentrations were slightly higher in fetuses of fasted mothers. Isoleucine concentrations also showed an increase, although there was no change

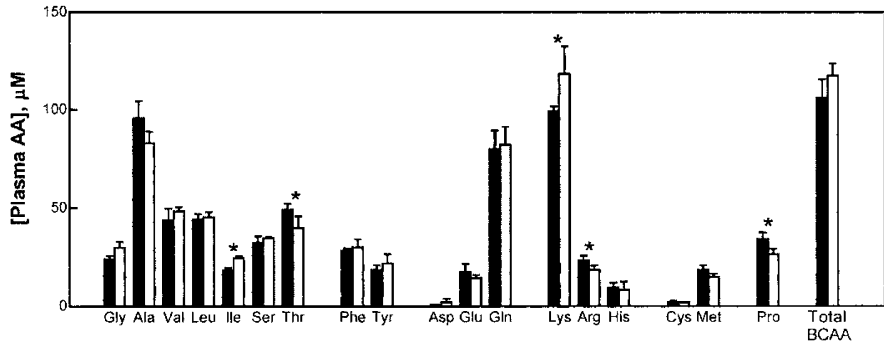

Figure 3. The effects of maternal fasting on fetal plasma amino acids. Pooled plasma from each of four control litters (solid bars) and four litters from fasted mothers (unfilled bars) were analyzed. Data are shown as mean $+1 \mathrm{SD}$. The final pair of bars shows data for total branched-chain amino acids (BCAA). *Significant difference ( $p<0.05$ by unpaired $t$ test) between the control and maternal fasting group for individual amino acids.

in the other branched-chain amino acid levels or in the total branched-chain amino acid concentration.

Based on the above, we focused on the effects of altered leucine, arginine, and glutamine concentrations on E19 hepatocyte proliferation in culture. Glucose was also studied, based on our previous studies demonstrating fetal hypoglycemia in the maternal starvation model (see "Discussion"). We first performed studies in which reductions in the concentrations of these nutrients were examined for an effect on E19 fetal hepatocyte incorporation of ${ }^{3} \mathrm{H}$-thymidine into DNA. Complete removal of glucose from the culture medium had no effect on DNA synthesis (data not shown). However, decreases in the concentrations of leucine or arginine reduced DNA synthesis (Fig. 4). The former showed a graded dose-response whereas the latter showed a threshold effect when the concentration was reduced from $5 \%(0.083 \mathrm{mM})$ to $1 \%(0.016 \mathrm{mM})$ of the concentration in standard MEM $\alpha$. As expected, reductions in glutamine concentration had no effect on DNA synthesis (Fig. 4).

The changes in DNA synthesis seen with altered nutrient concentration could not be definitively assigned to G1 growth arrest by measuring ${ }^{3} \mathrm{H}$-thymidine incorporation alone, so additional studies were performed. To measure BrdU incorporation into DNA, cells were exposed to media containing various nutrient concentrations for $18 \mathrm{~h}$. BrdU was then added to the media for an additional $6 \mathrm{~h}$. Immunofluorescent microscopy was used to obtain a nuclear labeling index. Results (Fig. 5) confirmed that elimination of glucose from the culture media had no effect on DNA synthesis. Significant decreases in the BrdU labeling index were seen with reduced leucine and arginine, but not glutamine. However, the degree of inhibition of DNA synthesis at low leucine and arginine concentrations was, in general, less extreme than that seen in the ${ }^{3} \mathrm{H}$-thymidine incorporation experiments, suggesting a possible loss of cell viability. Accordingly, we assessed effects on apoptosis using TUNEL staining. Results (Fig. 6) showed a modest increase in apoptosis in the presence of $10 \%$ leucine or $0 \%$ arginine. Elimination of leucine from the media was associated with a marked increase in the number of apoptotic cells, whereas $10 \%$ arginine showed no increase over control. Elimination of glutamine or glucose from the media did not result in an increase in TUNEL positive cells. The TUNEL staining results were 


\section{${ }^{3} \mathrm{H}$-Thymidine Uptake (cpm/mg protein)}
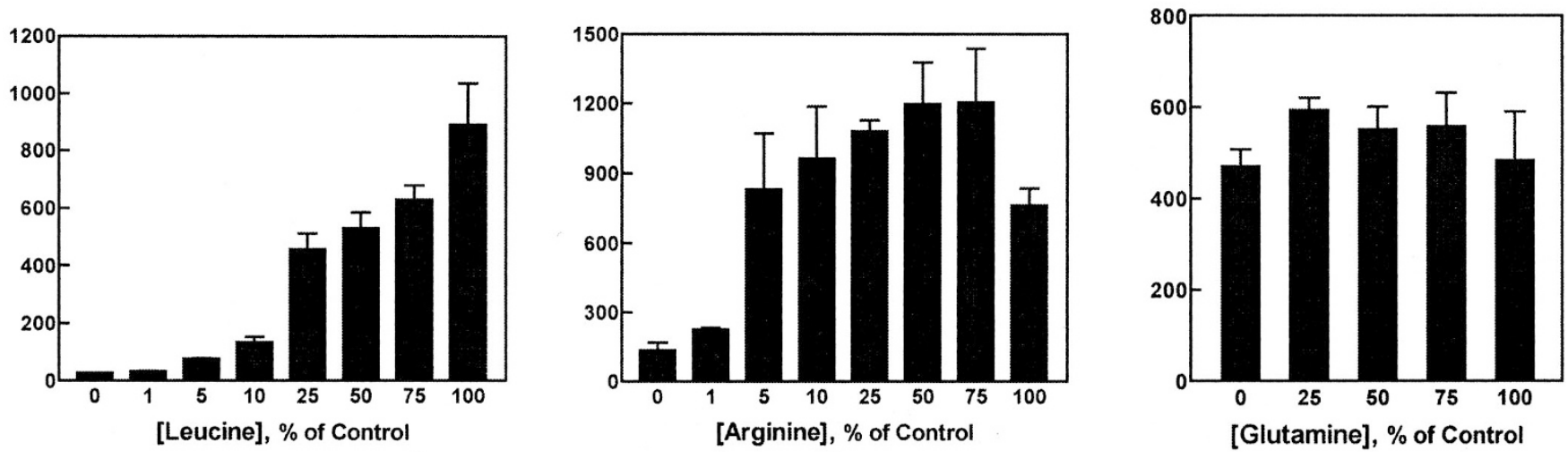

Figure 4. The effects of altered amino acid concentrations on DNA synthesis by cultured fetal hepatocytes. Hepatocytes isolated on E19 were cultured in the presence of varying concentrations of leucine, arginine, or glutamine. DNA synthesis was measured as the incorporation of ${ }^{3} \mathrm{H}$-thymidine into DNA, normalized per unit lysate protein. Data represent the mean $+1 \mathrm{SD}$ of three wells per condition. Amino acid concentrations are shown as a percentage of the concentrations in standard MEM $\alpha$. Similar results were obtained in two additional experiments.

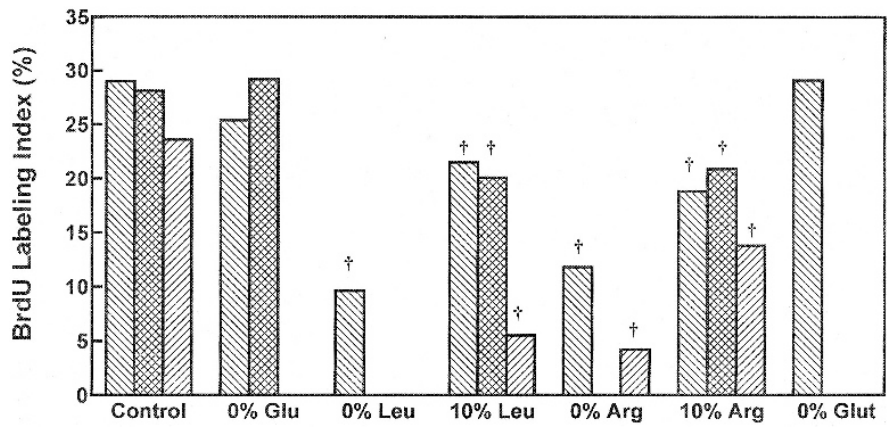

Figure 5. The effects of varying glucose (Glu) or amino acid concentrations on DNA synthesis by cultured fetal hepatocytes. Hepatocytes isolated on E19 were cultured in the presence of varying concentrations of glucose, leucine, arginine, or glutamine. DNA synthesis was assessed by the immunofluorescent detection of BrdU. Results, expressed as nuclear labeling indices, of three independent experiments are represented by the three bar fill patterns. $†$ Results outside of the $95 \%$ confidence limits for the corresponding control group.

confirmed using a dual fluorescent dye method to assess cell viability (data not shown). Again, marked loss of viability was seen with $0 \%$ leucine, whereas $10 \%$ leucine and $0 \%$ arginine showed modest reductions in viability. The other conditions were not associated with reduced viability.

We went on to examine the cell cycle regulation that could account for the observed changes in DNA synthesis. Exposure of E19 hepatocytes to media containing 10\% leucine or $20 \mathrm{nM}$ rapamycin for $24 \mathrm{~h}$ showed no effect on cyclin E content (Fig. 7) or cyclin D3 content (not shown). Cyclin D1 could not be detected. Although no change in cyclin E content was observed, we considered the possibility that cyclin E-dependent kinase activity might be affected. Immunoprecipitation kinase assays showed that both leucine limitation and rapamycin produced an inhibition of cyclin E-associated kinase activity (Fig. 7).

Parallel studies were performed to examine the effects of reduced arginine availability on E19 hepatocyte G1 control. Similar to the results obtained with leucine restriction and

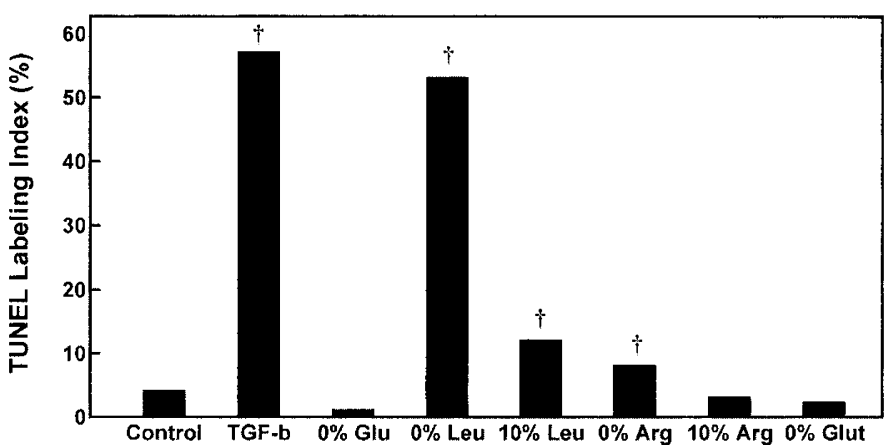

Figure 6. The induction of apoptosis in cultured E19 hepatocytes in response to altered nutrient concentration. Cells were cultured under the conditions used for the experiments shown in Figure 5. In addition, transforming growth factor- $\beta(T G F-b)$ was used as a positive control for the induction of fetal hepatocyte apoptosis. After $24 \mathrm{~h}$, the cells were fixed and analyzed by immunofluorescent TUNEL staining. Three high-power fields per condition were counted to obtain a nuclear labeling index. Over the course of two additional experiments, all of the amino acid results were reproduced at least once. $†$ Results outside of the $95 \%$ confidence limits for the corresponding control group.

rapamycin, reduction in arginine concentration in the culture media was associated with a dose-dependent inhibition of cyclin E-associated kinase activity without any reduction in cyclin E content (Fig. 8A). Western immunoblotting for CDK2 (Fig. $8 B$ ), the cognate kinase for cyclin E, showed a modest decrease in total content with arginine reduction. However, the amount of CDK2 that co-immunoprecipitated with cyclin E was unaffected. Western immunoblot analysis of the phosphorylation state of the cell cycle regulator $\mathrm{Rb}$ was used to confirm a selective effect on cyclin E-dependent kinase activity. Results (Fig. 8C) showed loss of Rb phosphorylation at the cyclin $\mathrm{E}$-dependent site, but not the cyclin $\mathrm{D}$-dependent site, in response to reduced arginine, reduced leucine, and rapamycin.

To confirm that reduction of culture media arginine concentration affects signaling through mTOR, we examined two downstream signaling events in the mTOR pathway. De- 


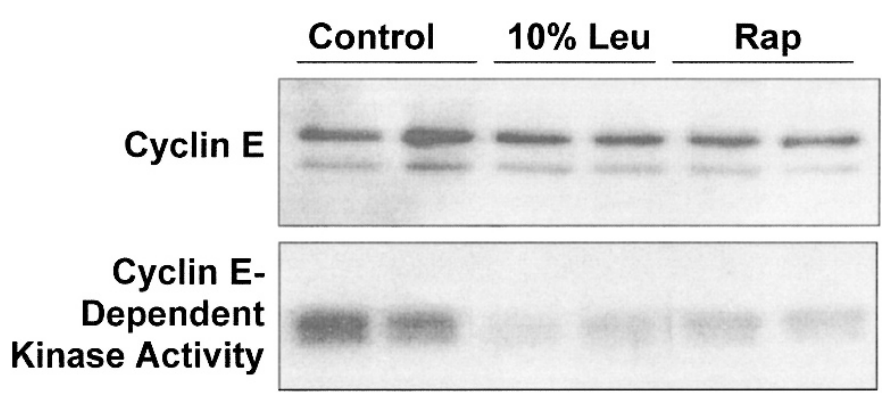

Figure 7. Cyclin E content and associated kinase activity in fetal hepatocytes exposed to reduced leucine or rapamycin. E19 hepatocytes were cultured for $24 \mathrm{~h}$ in the presence of $10 \%$ leucine or $20 \mathrm{nM}$ rapamycin. At the end of that time, cell lysates were prepared and analyzed for cyclin E content (Western blot, upper panel) or for cyclin E-associated kinase activity (autoradiogram, lower panel). Duplicate samples were analyzed for each condition.

creased arginine produced a dose-dependent decline in S6 phosphorylation, which could not be accounted for by a change in S6 content (Fig. 9A). Reduced arginine availability also resulted in a shift in the phosphorylation of eIF4E-associated 4E-BP1 from its phosphorylated $\beta$ form to its hypophosphorylated $\alpha$ form (Fig. 9B). Both effects were also seen with 20 nM rapamycin.

\section{DISCUSSION}

The overall aim of the present studies was to examine the mechanisms by which restricted substrate availability to the fetus might alter fetal liver growth. The rat is a well-established animal model for studying the affects of maternal starvation. Girard et al. (4) performed the first rigorous characterization of this model, demonstrating that $48 \mathrm{~h}$ of starvation at the end of term was associated with decreased circulating maternal concentrations of glucose, lactate, pyruvate, and insulin, whereas maternal branched-chain amino acid, FFA, and ketoacid concentrations rose. We chose E19 as the developmental time point for our studies based on several factors. The first was that fetal hepatocyte mitogenic signaling at this point in development is insulin insensitive $(7,8,16)$ and mitogen-independent $(15,17)$, based on our previous observations. Secondly, we have shown previously that fetal hepatocytes undergo cell cycle arrest at term $(2,13)$, thereby preventing analysis of cell cycle control in more mature fetuses.

We first showed that maternal starvation for $48 \mathrm{~h}$ before delivery on E19 was associated a reduction in fetal liver weight, no apparent change in hepatocyte size, and a decrease in hepatocyte cell cycle activity. The latter was demonstrated by PCNA labeling index, which, as we have previously shown, correlates with the proliferation of cultured hepatocytes from fetuses of different gestational ages (2). We have used the in vivo PCNA labeling index as an indicator of fetal hepatocyte cell cycle activity (13). Using flow cytometry on freshly isolated hepatocytes, we were able to confirm that maternal fasting was associated with fetal hepatocyte G1, as opposed to G2, growth arrest.

An analysis of G1 cell cycle components using fetal liver homogenates did not demonstrate an effect of maternal fasting. We considered it likely that the cell cycle effects in vivo were too subtle to be detected as changes in these outcome measures. Therefore, we focused our experiments on analysis of the effects of nutrient concentration on proliferation in cultured E19 hepatocytes. The candidate nutrients we considered for a role in the control of hepatocyte proliferation included glucose and amino acids. We have previously shown that maternal starvation for $2 \mathrm{~d}$ before delivery is associated with an approximately $25 \%$ reduction in fetal plasma glucose concentration (11). Although circulating amino acid concentrations in rat models of intrauterine growth restriction have been examined by a number of investigators, we were unable to find data pertaining to our specific experimental conditions ( $48 \mathrm{~h}$ maternal starvation before cesarean delivery on E19). The results we obtained indicated that reductions in fetal plasma branchedchain amino acid concentrations were not associated with our model. We nonetheless studied leucine for its established role in regulating the activity of the key nutrient sensing kinase, mTOR (18). Glutamine, a nonessential amino acid, was used as a control. Arginine was chosen for the following reasons. First, its concentration declined in fetuses of fasted mothers. Secondly, arginine is an essential amino acid under conditions of rapid protein accretion $(19,20)$. Third, arginine may function as a regulator of hepatocyte amino acid transport (21).

Our in vitro studies showed that complete removal of glucose from the culture medium had no effect on DNA synthesis, but that decreases in the concentrations of leucine or arginine reduced DNA synthesis. Whereas leucine showed a graded dose-response, reductions in arginine showed a threshold effect. We interpreted this as indicating a point at which endogenous hepatocyte arginine production could no longer support protein synthesis. However, in the absence of a direct measurement of protein synthetic rates, this remains speculative. Alternatively, this may be a phenomenon that is a result of the direct regulation of mTOR by arginine.

In addition to effects on DNA synthesis, our data suggested a loss of cell viability under conditions of nutrient restriction that might be secondary to apoptosis. Our results indicated that restriction of leucine or arginine, but not glutamine or glucose, was associated with a combined inhibition of DNA synthesis and induction of apoptosis. However, modest nutrient restriction (10\% leucine or 5-10\% percent arginine) caused a significant reduction in DNA synthesis without induction of apoptosis. These results are consistent with several published observations in nonhepatic cell systems. Arginine restriction has been shown to produce growth arrest in fibroblasts through the inhibition of CDK4 expression (22). It has also been shown, along with leucine restriction, to induce apoptosis in vascular smooth muscle cells (23). Others have shown that increased arginine availability can protect against apoptosis induced by exposure of $\beta$-cells to low glucose (24), or by exposure of HL-60 cells to a variety of apoptosis-inducing agents (25).

Examination of the cell cycle events that could account for G1 growth arrest in the presence of low leucine or arginine concentration revealed a central role for cyclin E-containing complexes. Western blotting to determine D-type cyclin content in E19 hepatocyte lysates has been problematic in that levels are very low. We were able to detect low but unaltered 
A

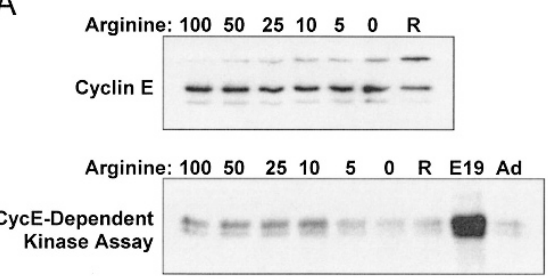

B
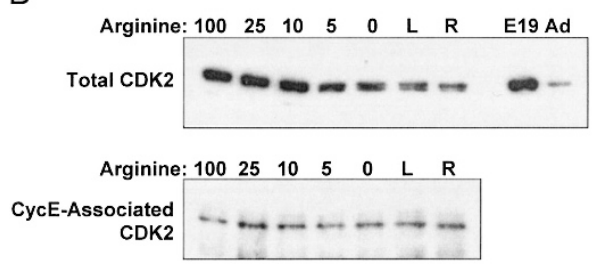

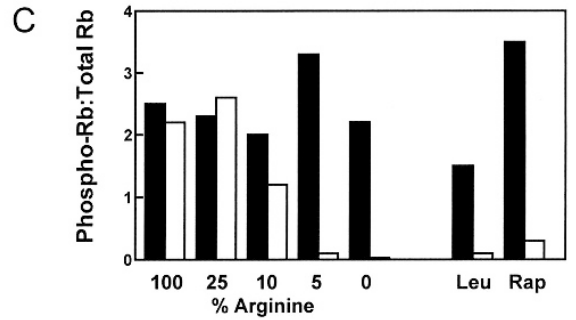

Figure 8. The cell cycle response of cultured fetal hepatocytes to reduced arginine concentration. E19 hepatocytes were cultured for $24 \mathrm{~h}$ in custom MEM $\alpha$ containing arginine at $100 \%$ to $0 \%$ of the standard concentration in MEM $\alpha$. Additional control conditions included $10 \%$ leucine (L or Leu) or $20 \mathrm{nM}$ rapamycin (R or Rap). Homogenates of fetal liver (E19) and adult liver (Ad) were also used as comparison samples for some analyses. Samples were analyzed for the following: Panel A shows analyses for cyclin E content and cyclin E-associated kinase activity. Panel B shows analyses for CDK2 content and cyclin E-associated CDK2. The latter was determined by immunoprecipitation with anti-cyclin $\mathrm{E}$ followed by Western blotting of the immunoprecipitates for CDK2. Panel $C$ shows the ratios of phospho- $\mathrm{Rb}$ to total $\mathrm{Rb}$ for two sets of Western blots. The first, shown by the solid bars, detected phosphorylation at Ser780, the cyclin D-dependent site on Rb. The second analysis, shown by the unfilled bars, detected phosphorylation at Ser807/811, the cyclin E-dependent site on Rb.

A

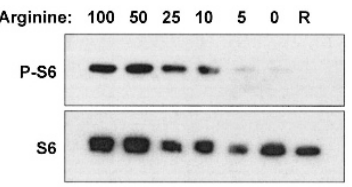

B

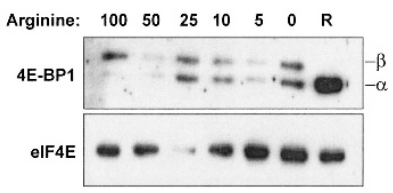

Figure 9. The effects of reduced arginine concentration on fetal hepatocyte translational control. (A) E19 hepatocytes were cultured in the presence of varying arginine concentrations or $20 \mathrm{nM}$ rapamycin (R) for $24 \mathrm{~h}$. At the end of that time, cell lysates were prepared and analyzed by Western immunoblotting for phospho-S6 and total S6. $(B)$ The same lysates were subjected to affinity purification of the eIF4E complex using ${ }^{7}$ methyl-GTP-Sepharose 4B. The affinity-purified preparations were subjected to Western immunoblotting for eIF4E and 4E-BP1. The designations to the right of the 4E-BP1 immunoblot represent the hypophosphorylated $(\alpha)$ and hyperphosphorylated $(\beta)$ forms of $4 \mathrm{E}-\mathrm{BP} 1$ that bind to eIF4E.

levels of cyclin D3, consistent with our own unpublished observation that cyclin D3 is present at high levels in E19 liver relative to adult liver. More importantly, the finding that cyclin $\mathrm{E}$ was unaffected by leucine restriction or rapamycin is a strong indicator that cyclin D-dependent complexes are also unaffected. This is based on longstanding observations that place cyclin D upstream from cyclin E transcriptional regulation during the G1 phase of the cell cycle (26). Leucine restriction, arginine restriction, and exposure to rapamycin were all associated with a decrease in fetal hepatocyte CDK2 content. However, these results are consistent with the possibility that CDK2 content parallels cell proliferation, a conclusion that is consistent with the reduced CDK2 seen in adult liver relative to fetal liver. It is unlikely that changes in CDK2 content are responsible for the observed changes in cyclin E-associated kinase activity, inasmuch as cyclin E-associated CDK2 was unaffected. However, direct measurement of cyclin E-associated kinase activity showed an effect of all three conditions. This was confirmed by analysis for $\mathrm{Rb}$ phosphorylation at sites Ser780 and Ser807/811, which have been shown to be cyclin D dependent and cyclin E dependent, respectively $(27,28)$. Results were consistent with a selective inhibition of cyclin E-dependent kinase activity.

Further experiments supported the hypothesis that the arginine-mediated effects might be due to a change in mTOR signaling. Two signaling events that require mTOR were examined, phosphorylation of ribosomal protein S6 and the regulator of the initiation factor eIF4E, 4E-BP1 (29). The

mTOR dependence of both was readily demonstrated by adding rapamycin to the fetal hepatocyte cultures. The hypophosphorylation of both S6 and 4E-BP1 that was induced by arginine restriction was consistent with inhibition of mTOR signaling. The arginine dose-response for the two effects did not coincide, perhaps owing to the fact that mTOR signaling is complex and not a simple function of the control of mTOR kinase activity (9).

Our results are consistent with the hypothesis that maternal starvation induces fetal hepatocyte G1 growth arrest secondary to loss of kinase activity in cyclin E-containing complexes. These effects may be mediated by the nutrient sensor, mTOR. Several aspects of the results deserve comment. The first is that our findings may represent a hormone- and growth factorindependent mechanism for the control of the fetal hepatocyte cell cycle. This conclusion is consistent with our previous studies that have supported the hypothesis that regulation of fetal hepatocyte proliferation in the late gestation rat is by and large independent of the hormonal- and growth factorresponsive signaling pathways that operate in the adult animal $(1,15-17)$. A second finding in these studies, one that is novel, is that the amino acid arginine can modulate signaling through mTOR in hepatocytes. We found that restriction of arginine availability in vitro induced changes in cell cycle control and signaling to the ribosome that paralleled those that occurred in response to both leucine restriction and rapamycin. Arginine is an essential amino acid in fetal and newborn animals (20). It behaves as an essential amino acid in conditions of rapid liver growth such as liver regeneration after partial hepatectomy (30). Our results are consistent with either a specific mechanism by which arginine signals to mTOR, or a more general mechanism whereby mTOR signaling responds to factors that limit protein synthesis. In either case, the known mechanisms for mTOR regulation (31) do not account for the effects of arginine restriction that we observed.

Another finding is that inhibition of signaling through mTOR resulted in a selective inhibition of cyclin E-associated kinase activity. Signaling by D-type cyclins appeared to be unaffected. The inhibition of cyclin E-associated kinase activity could not be accounted for by a decrease in cyclin E content or CDK2 content. It is possible that induction of a CKI may be involved. However, the CKI that inhibit cyclin E-dependent 
kinases are also active with cyclin D-containing complexes (32). Other potential mechanisms might involve posttranslational modification of cyclin E, CDK2, or both. Elucidation of the precise mechanism that accounts for these observations may have implications not only for the regulation of fetal hepatocyte proliferation but also for other conditions in which the growth of hepatic cells come under the influence of altered nutrient availability.

Acknowledgments. The authors thank Dr. Mary G. Ampola for performing the plasma amino acid analyses.

\section{REFERENCES}

1. Curran TR Jr, Bahner RI Jr, Oh W, Gruppuso PA 1993 Mitogen-independent DNA synthesis by fetal rat hepatocytes in primary culture. Exp Cell Res 209:53-57

2. Gruppuso PA, Awad M, Bienieki TC, Boylan JM, Fernando S, Faris RA 1997 Modulation of mitogen-independent hepatocyte proliferation during the perinatal period in the rat. In Vitro Cell Dev Biol Anim 33:562-568

3. Wigglesworth JS 1964 Experimental growth retardation in the foetal rat. J Pathol Bacteriol 88:1-13

4. Girard JR, Ferre P, Gilbert M, Kervran A, Assan R, Marliss EB 1977 Fetal metabolic response to maternal fasting in the rat. Am J Physiol 232:E456-E463

5. Gruppuso PA, Migliori R, Susa JB, Schwartz R 1981 Chronic maternal hyperinsulinemia and hypoglycemia. A model for experimental intrauterine growth retardation. Biol Neonate 40:113-120

6. Cornblath M, Schwartz R 1991 Disorders of Carbohydrate Metabolism in Infancy. Blackwell Scientific Publications, Boston, pp 12-17

7. Anand P, Boylan JM, Ou Y, Gruppuso PA 2002 Insulin signaling during perinatal liver development in the rat. Am J Physiol Endocrinol 283:E844-E852

8. Khamzina L, Gruppuso PA, Wands JR 2003 Insulin signaling through insulin receptor substrate 1 and 2 in normal liver development. Gastroenterology 125:572-585

9. Schmelzle T, Hall MN 2000 TOR, a central controller of cell growth. Cell 103:253262

10. Rohde J, Heitman J, Cardenas ME 2001 The TOR kinases link nutrient sensing to cell growth. J Biol Chem 276:9583-9586

11. Gruppuso PA, Brautigan DL 1989 Induction of hepatic glycogenesis in the fetal rat. Am J Physiol 256:E49-E54

12. Slocum RH, Cummings JG 1991 Amino acid analysis of physiological samples. In: Hommes FA (ed) Techniques in Diagnostic Human Biochemical Genetics. WileyLiss, New York, pp 87-126

13. Awad MM, Gruppuso PA 2000 Cell cycle control during liver development in the rat: evidence indicating a role for cyclin D1 post-transcriptional regulation. Cell Growth Differ 11:325-334
14. Gruppuso PA, Bienieki TC, Faris RA 1999 The relationship between differentiation and proliferation in late gestation fetal rat hepatocytes. Pediatr Res 46:14-19

15. Boylan JM, Anand P, Gruppuso PA 2001 Ribosomal protein S6 phosphorylation and function during late gestation liver development in the rat. J Biol Chem 276:4445744463

16. Boylan JM, Gruppuso PA 1996 A comparative study of the hepatic mitogen-activated protein kinase and Jun- $\mathrm{NH}_{2}$-terminal kinase pathways in the late-gestation fetal rat. Cell Growth Differ 7:1261-1269

17. Boylan JM, Gruppuso PA 1998 Uncoupling of hepatic, epidermal growth factormediated mitogen-activated protein kinase activation in the fetal rat. J Biol Chem 273:3784-3790

18. Raught B, Gingras AC, Sonenberg N 2001 The target of rapamycin (TOR) proteins Proc Natl Acad Sci U S A 98:7037-7044

19. Wheatley DN, Campbell E 2002 Arginine catabolism, liver extracts and cancer Pathol Oncol Res 8:18-25

20. Wu G, Meininger CJ, Knabe DA, Bazer FW, Rhoads JM 2000 Arginine nutrition in development, health and disease. Curr Opin Clin Nutr Metab Care 3:59-66

21. Campbell WA, Sah DE, Medina MM, Albina JE, Coleman WB, Thompson NL 2000 TA1/LAT-1/CD98 light chain and system L activity, but not 4F2/CD98 heavy chain, respond to arginine availability in rat hepatic cells. Loss of response in tumor cells J Biol Chem 275:5347-5354

22. Lamb J, Wheatley DN 2000 Single amino acid (arginine) deprivation induces G1 arrest associated with inhibition of cdk4 expression in cultured human diploid fibroblasts. Exp Cell Res 255:238-249

23. Earle KA, Pancholi S, Vernon P, Yudkin JS 1998 Amino acid depletion modulates vascular endothelial growth factor production during the life span of human vascular smooth muscle cells. J Cell Physiol 176:359-364

24. Van de Casteele M, Kefas BA, Cai Y, Heimberg H, Scott DK, Henquin JC, Pipeleer D, Jonas JC 2003 Prolonged culture in low glucose induces apoptosis of rat pancreatic beta-cells through induction of c-myc. Biochem Biophys Res Commun 312:937-944

25. Sakagami H, Satoh M, Yokote Y, Takano H, Takahama M, Kochi M, Akahane K 1998 Amino acid utilization during cell growth and apoptosis induction. Anticancer Res 18:4303-4306

26. Sherr CJ, Kato J, Quelle DE, Matsuoka M, Roussel MF 1994 D-type cyclins and their cyclin-dependent kinases: G1 phase integrators of the mitogenic response. Cold Spring Harb Symp Quant Biol 59:11-19

27. Rickheim DG, Nelsen CJ, Fassett JT, Timchenko NA, Hansen LK, Albrecht JH 2002 Differential regulation of cyclins D1 and D3 in hepatocyte proliferation. Hepatology 36:30-38

28. Jinno S, Hung SC, Yamamoto H, Lin J, Nagata A, Okayama H 1999 Oncogenic stimulation recruits cyclin-dependent kinase in the cell cycle start in rat fibroblast. Proc Natl Acad Sci U S A 96:13197-13202

29. Proud CG 2002 Regulation of mammalian translation factors by nutrients. Eur J Biochem 269:5338-5349

30. Lorenzi M, De Martino A, Carlucci F, Tabucchi A, Porcelli B, Pizzichini M, Marinello E, Pagani R 1993 Nitrogen metabolism during liver regeneration. Biochim Biophys Acta 1157:9-14

31. Gingras AC, Raught B, Sonenberg N 2001 Control of translation by the target of rapamycin proteins. Prog Mol Subcell Biol 27:143-174

32. Sherr CJ, Roberts JM 1995 Inhibitors of mammalian G1 cyclin-dependent kinases Genes Dev 9:1149-1163 